MATHEMATICS OF COMPUTATION

Volume 70, Number 235, Pages 1183-1194

S $0025-5718(00) 01268-0$

Article electronically published on April 13, 2000

\title{
ON NONOSCILLATING INTEGRALS FOR COMPUTING INHOMOGENEOUS AIRY FUNCTIONS
}

\author{
AMPARO GIL, JAVIER SEGURA, AND NICO M. TEMME
}

\begin{abstract}
Integral representations are considered of solutions of the inhomogeneous Airy differential equation $w^{\prime \prime}-z w= \pm 1 / \pi$. The solutions of these equations are also known as Scorer functions. Certain functional relations for these functions are used to confine the discussion to one function and to a certain sector in the complex plane. By using steepest descent methods from asymptotics, the standard integral representations of the Scorer functions are modified in order to obtain nonoscillating integrals for complex values of $z$. In this way stable representations for numerical evaluations of the functions are obtained. The methods are illustrated with numerical results.
\end{abstract}

\section{INTRODUCTION}

Airy functions are solutions of the differential equation

$$
\frac{d^{2} w}{d z^{2}}-z w=0
$$

Two linearly independent solutions that are real for real values of $z$ are denoted by $\operatorname{Ai}(z)$ and $\operatorname{Bi}(z)$. They have the integral representations

$$
\begin{aligned}
& \operatorname{Ai}(z)=\frac{1}{\pi} \int_{0}^{\infty} \cos \left(z t+\frac{1}{3} t^{3}\right) d t \\
& \operatorname{Bi}(z)=\frac{1}{\pi} \int_{0}^{\infty} \sin \left(z t+\frac{1}{3} t^{3}\right) d t+\frac{1}{\pi} \int_{0}^{\infty} e^{z t-\frac{1}{3} t^{3}} d t,
\end{aligned}
$$

where we assume that $z$ is real.

In this paper we concentrate on so-called Scorer functions (7, 15]), which are particular solutions of the nonhomogeneous Airy differential equation. We have

$$
w^{\prime \prime}-z w=-1 / \pi, \quad \text { with solution } \quad \operatorname{Gi}(z)=\frac{1}{\pi} \int_{0}^{\infty} \sin \left(z t+\frac{1}{3} t^{3}\right) d t, \quad z \in \mathbb{R},
$$

and

$$
w^{\prime \prime}-z w=1 / \pi, \quad \text { with solution } \quad \operatorname{Hi}(z)=\frac{1}{\pi} \int_{0}^{\infty} e^{z t-\frac{1}{3} t^{3}} d t, \quad z \in \mathbb{C} .
$$

Received by the editor September 11, 1998 and, in revised form, April 27, 1999 and August 25, 1999.

2000 Mathematics Subject Classification. Primary 33C10, 41A60, 30E10 65D20.

Key words and phrases. Inhomogeneous Airy functions, Scorer functions, method of steepest descent, saddle point method, numerical computation of special functions.

(C)2000 American Mathematical Society 
Initial values are

$$
\begin{gathered}
\operatorname{Gi}(0)=\frac{1}{2} \operatorname{Hi}(0)=\frac{1}{3} \operatorname{Bi}(0)=\frac{1}{\sqrt{3}} \operatorname{Ai}(0)=\frac{1}{3^{7 / 6} \Gamma\left(\frac{2}{3}\right)}, \\
\operatorname{Gi}^{\prime}(0)=\frac{1}{2} \operatorname{Hi}^{\prime}(0)=\frac{1}{3} \operatorname{Bi}^{\prime}(0)=-\frac{1}{\sqrt{3}} \operatorname{Ai}^{\prime}(0)=\frac{1}{3^{5 / 6} \Gamma\left(\frac{1}{3}\right) .}
\end{gathered}
$$

From (1.2), (1.3) and (1.4) it follows that

$$
\operatorname{Gi}(z)+\operatorname{Hi}(z)=\operatorname{Bi}(z) .
$$

In the next section we give contour integrals from which representations of $\operatorname{Ai}(z), \operatorname{Bi}(z)$ and $\operatorname{Gi}(z)$ follow for complex values of $z$. Just like $\operatorname{Ai}(z)$ and $\operatorname{Bi}(z)$, the Scorer functions $\operatorname{Gi}(z)$ and $\operatorname{Hi}(z)$ are entire functions.

A survey on computational aspects of special functions, including information on Airy functions, can be found in [8]; [10] has a public web site that includes an extensive treatment of Scorer functions. For complex values of $z$ the Airy functions are available in the Bessel function algorithms of [2]; see also [3] and [14]. Computer algebra systems as Maple and Mathematica also have Airy functions available. The Scorer functions are considered in [9, where coefficients of Chebyshev expansions are given for real $z$. Asymptotic expansions for $\operatorname{Hi}(z)$ are given in [4] and [11. The paper by Scorer ([15]) gives tables to 7 decimals of $\operatorname{Hi}(-z)$ and $\operatorname{Gi}(z)$ for $0 \leq z \leq 10$.

Efficient algorithms for computing the Scorer functions in restricted domains of the complex plane can be based on Maclaurin series and asymptotic series. These domains can be bridged by using the differential equations or the integral representations.

In [5] methods were presented based on the differential equations, which are set up as boundary-value methods, providing stable algorithms for all values of $z$.

The purpose of this paper is to give stable integral representations for $\operatorname{Gi}(z)$ and $\operatorname{Hi}(z)$. We modify the integrals in (1.3) and (1.4) by using methods from asymptotics. As in [5], the resulting integrals can be used for any value of the complex parameter $z$. We also indicate how similar methods can be used for the Airy function itself.

\section{Asymptotic properties of the Airy and Scorer functions}

We need a few properties of the Airy and Scorer functions. More information can be found in [1], 10], [11] and [17]. In particular [11, Chapter 11, discusses numerically satisfactory solutions of the differential equations for $\operatorname{Gi}(z)$ and $\operatorname{Hi}(z)$. The asymptotic properties of the Airy and Scorer functions are important in this discussion.

We write, as in [11,

$$
\operatorname{Ai}_{0}(z)=\operatorname{Ai}(z), \quad \operatorname{Ai}_{1}(z)=\operatorname{Ai}\left(e^{-2 \pi i / 3} z\right), \quad \operatorname{Ai}_{-1}(z)=\operatorname{Ai}\left(e^{2 \pi i / 3} z\right) .
$$

We have the representations

$$
\operatorname{Ai}_{j}(z)=\frac{e^{2 j \pi i / 3}}{2 \pi i} \int_{\mathcal{C}_{-j}} e^{-z t+\frac{1}{3} t^{3}} d t, \quad j=0, \pm 1,
$$



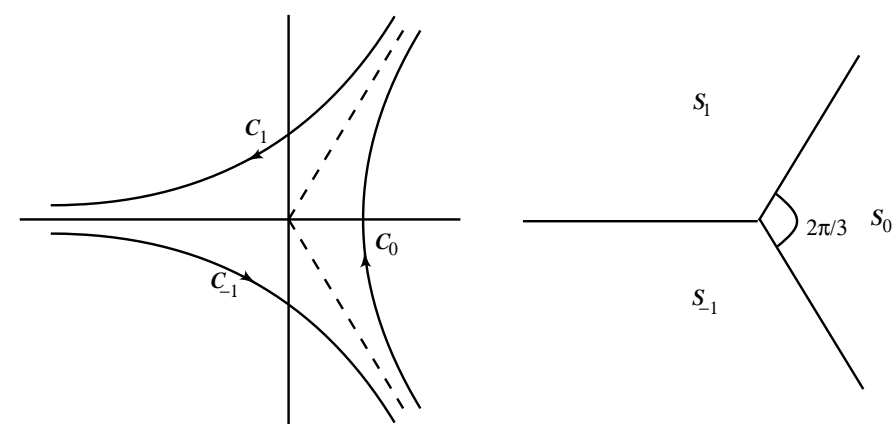

FiguRE 2.1. Three contours $\mathcal{C}_{j}$ of integration for the Airy integrals in (2.2), and sectors $S_{j}$ where the $\mathrm{Ai}_{j}(z)$ are recessive.

where the contours $\mathcal{C}_{j}$ are given in Figure 2.1. Because $\int_{\mathcal{C}_{0} \cup \mathcal{C}_{1} \cup \mathcal{C}_{-1}} e^{-z t+\frac{1}{3} t^{3}} d t=0$, we have the following linear combination of three solutions of (1.1):

$$
\mathrm{Ai}(z)+e^{-2 \pi i / 3} \mathrm{Ai}_{1}(z)+e^{2 \pi i / 3} \mathrm{Ai}_{-1}(z)=0 .
$$

The first integral in (1.2) follows from deforming the contour $\mathcal{C}_{0}$ in (2.2) into the imaginary axis. The function $\operatorname{Bi}(z)$ can be written as

$$
\operatorname{Bi}(z)=e^{\pi i / 6} \mathrm{Ai}_{-1}(z)+e^{-\pi i / 6} \mathrm{Ai}_{1}(z)
$$

and the second representation in (1.2) follows by deforming the contour $\mathcal{C}_{1}$ into the positive imaginary axis and $(-\infty, 0]$, and the contour $\mathcal{C}_{-1}$ into $(-\infty, 0]$ and the negative imaginary axis.

The function $\operatorname{Ai}_{j}(z)$ is recessive at infinity in the sector $S_{j}, j=0, \pm 1$, the function being exponentially small at infinity along any ray interior to this sector. On the other hand, $\operatorname{Ai}_{j}(z)$ is dominant at infinity in the sectors $S_{j-1}$ and $S_{j+1}$ (the suffix $j$ is enumerated modulo 3 ), and is exponentially large at infinity along the rays interior to these sectors. $\operatorname{Bi}(z)$ is dominant at infinity within all three sectors $S_{j}$. A pair of Airy functions comprises a numerically satisfactory pair at infinity within a sector if only one function is dominant. For example, the pair $\{\operatorname{Ai}(z), \operatorname{Bi}(z)\}$ comprises such a pair only in $S_{0}$ (and on the negative real axis, where neither of the two is dominant, but where the phases in their oscillations differ by $\left.\frac{1}{2} \pi\right)$.

If one of the functions $\operatorname{Gi}(z), \operatorname{Hi}(z)$ is computed, we may use (1.6) to compute the other one (we assume in this paper that $\operatorname{Bi}(z)$ and all other Airy functions are available), but we need to know if (1.6) is numerically stable for that computation. For example, because $\operatorname{Gi}(z)$ is only of algebraic growth in $S_{0}$ (as we will see soon), we cannot compute $\operatorname{Gi}(z)$ from (1.6) in $S_{0}$.

For the Scorer functions we have the following asymptotic expansions (cf. [11, 431-432):

$$
\begin{gathered}
\operatorname{Hi}(z) \sim-\frac{1}{\pi z}\left[1+\frac{1}{z^{3}} \sum_{s=0}^{\infty} \frac{(3 s+2) !}{s !\left(3 z^{3}\right)^{s}}\right], \quad z \rightarrow \infty, \quad|\operatorname{ph}(-z)| \leq \frac{2}{3} \pi-\delta, \\
\operatorname{Gi}(z) \sim \frac{1}{\pi z}\left[1+\frac{1}{z^{3}} \sum_{s=0}^{\infty} \frac{(3 s+2) !}{s !\left(3 z^{3}\right)^{s}}\right], \quad z \rightarrow \infty, \quad|\operatorname{ph} z| \leq \frac{1}{3} \pi-\delta,
\end{gathered}
$$


$\delta$ being an arbitrary positive constant. For (2.6) the domain for ph $z$ is not given in [11], but it follows from the same method mentioned for $\operatorname{Hi}(z)$ in [11], p. 432. In other parts of the complex plane we cannot represent the Scorer functions by a single expansion with leading term $\mathcal{O}(1 / z)$.

From the results in (2.5) and (2.6) and the dominant asymptotic behavior of $\operatorname{Bi}(z)$ in all sectors $S_{j}, j=0, \pm 1$, we conclude (cf. (1.6) ) that $\operatorname{Bi}(z)$ is a dominant term for $\operatorname{Hi}(z)$ in $S_{0}$ and for $\operatorname{Gi}(z)$ in $S_{1} \cup S_{-1}$. It follows that we need algorithms for the computation of $\operatorname{Gi}(z)$ for $z \in S_{0}$ and for $\operatorname{Hi}(z)$ for $z \in S_{1} \cup S_{-1}$ (where the asymptotic expansions (2.5) and (2.6) are valid). The relation in (1.6) can be used for computing the functions in the complements of these domains (where the functions have the dominant behaviour of $\operatorname{Bi}(z))$.

A further reduction of domains follows from the connection formula 1

$$
\operatorname{Hi}(z)=e^{ \pm 2 \pi i / 3} \operatorname{Hi}\left(z e^{ \pm 2 \pi i / 3}\right)+2 e^{\mp \pi i / 6} \operatorname{Ai}\left(z e^{\mp 2 \pi i / 3}\right) .
$$

To prove this relation, observe that the first term in the right-hand side satisfies the differential equation for $\operatorname{Hi}(z)$, and that, hence, that term can be written as a linear combination of $\operatorname{Hi}(z)$ and solutions of the homogeneous equation; the initial values in 1.5 can be used to identify these solutions.

For example, we can use (2.7) with the upper signs for $z$ in the sector $\frac{1}{3} \pi<$ ph $z<\frac{2}{3} \pi$. Then, $\operatorname{Hi}(z)$ can be expressed in terms of Hi in the sector $-\pi<\mathrm{ph} z<$ $-\frac{2}{3} \pi$ plus an Airy function in the sector $-\pi / 3<\mathrm{ph} z<0$. We see that both functions in the right-hand side of (2.7) are not dominant in the respective sectors, and, hence, this representation is stable.

A similar connection formula for $\operatorname{Gi}(z)$ reads

$$
\operatorname{Gi}(z)=e^{ \pm 2 \pi i / 3} \mathrm{Gi}\left(z e^{ \pm 2 \pi i / 3}\right)+e^{\mp \pi i / 6} \mathrm{Ai}\left(z e^{\mp 2 \pi i / 3}\right) .
$$

This formula is of no use in the sector $S_{0}$ because both functions in the right-hand side are dominant, whereas $\operatorname{Gi}(z)$ is of algebraic growth at infinity within $S_{0}$. A better formula for $z \in S_{0}$ follows from combining (1.6), 2.4) and (2.7) (the latter twice, with upper and lower signs). This gives

$$
\operatorname{Gi}(z)=-\frac{1}{2}\left[e^{2 \pi i / 3} \mathrm{Hi}\left(z e^{2 \pi i / 3}\right)+e^{-2 \pi i / 3} \mathrm{Hi}\left(z e^{-2 \pi i / 3}\right)\right] .
$$

For $z \in S_{0}$ the arguments of the $\mathrm{Hi}$-functions are in $S_{ \pm 1}$, where these functions have expansions that follow from (2.5).

Because of the equalities

$$
\operatorname{Hi}(x-i y)=\overline{\operatorname{Hi}(x+i y)} \text { and } \operatorname{Gi}(x-i y)=\overline{\operatorname{Gi}(x+i y)}
$$

we can concentrate on nonnegative values of the imaginary part $y$ of the argument $z=x+i y$.

Conclusion. The principal domain of interest for the Scorer functions is the sector $\frac{2}{3} \pi \leq \operatorname{ph} z \leq \pi$, where we concentrate on $\operatorname{Hi}(z)$. For $z$ in other sectors, and for $\mathrm{Gi}(z)$, the relations (1.6), (2.7) and (2.8) are numerically stable for the particular cases.

For a summary of the results of this section and algorithms we refer to Section 4 .

\footnotetext{
${ }^{1}$ With thanks to the referee.
} 


\section{The CONSTRUCTION OF NONOSCILlATING INTEGRALS}

We modify the integral in (1.4) such that stable algorithms can be based on the new integral for $z$ in the sector $\frac{2}{3} \pi \leq$ ph $z \leq \pi$.

We write

$$
\phi(t)=\frac{1}{3} t^{3}-z t, \quad t=u+i v, \quad z=x+i y .
$$

Then the real and imaginary parts of $\phi(t)=\phi_{r}(u, v)+i \phi_{i}(u, v)$ are given by

$$
\begin{aligned}
& \phi_{r}(u, v)=\frac{1}{3} u^{3}-u v^{2}-x u+y v, \\
& \phi_{i}(u, v)=u^{2} v-\frac{1}{3} v^{3}-x v-y u .
\end{aligned}
$$

We are interested in a path in the complex $t$-plane on which $\phi_{i}(u, v)$ is a constant, and the path should start at the origin, as the integral in (1.4). Such a path is defined by the equation

$$
u^{2} v-\frac{1}{3} v^{3}-x v-y u=0,
$$

and we need real solutions of this equation.

As summarized at the end of Section 2, we need to consider $z$-values satisfying $2 \pi / 3 \leq$ ph $z \leq \pi$. If $z<0$ we can integrate along the positive $t$-axis. For other values of ph $z$ in the present range the path of integration will be deformed into a curve $\mathcal{C}$ that is defined by (3.3) and that runs from the origin to $+\infty$; see Figure 3.1 .
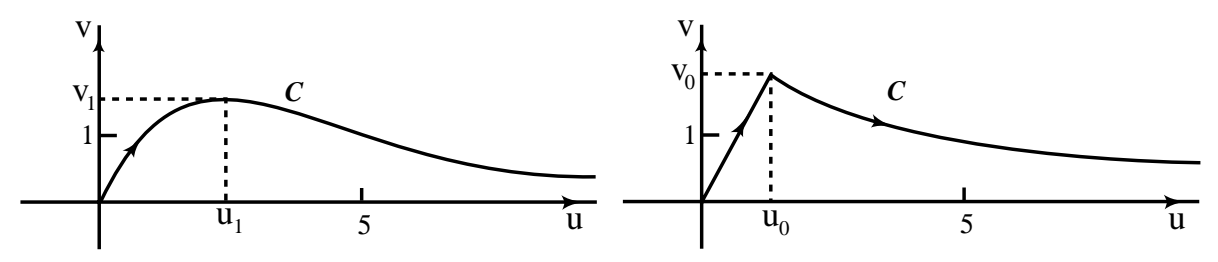

Figure 3.1. The contour $\mathcal{C}$ is defined by (3.4). The left figure is for $z$ inside the sector $2 \pi / 3 \leq \mathrm{ph} z \leq \pi$, and the curve is defined by (3.4). If ph $z=2 \pi / 3$, the curve in the right figure is defined by (3.8).

We solve the cubic equation (3.3) for $v$. The solution that passes through the origin can be written in the form

$$
v(u)=2 \sqrt{Q} \sin \frac{1}{3} \theta
$$

where

$$
\theta=\arcsin \frac{P}{\sqrt{Q^{3}}} \in\left[0, \frac{1}{2} \pi\right], \quad P=\frac{3}{2} y u, \quad Q=u^{2}-x,
$$

with $u \geq 0, x<0,3 x^{2}>y^{2}$.

To show this, we introduce $t=v /(2 \sqrt{Q})$. Then $(3.3)$ can be written in the form

$$
4 t^{3}-3 t=-\frac{P}{\sqrt{Q^{3}}} \text {. }
$$


It is not difficult to verify that, if $u \geq 0, x<0,3 x^{2}>y^{2}$, then the modulus of the right-hand side is not larger than unity. Replacing the left-hand side with $-\sin (3 \arcsin (t))$ gives the solution in (3.5).

It follows that

$$
\operatorname{Hi}(z)=\frac{1}{\pi} \int_{0}^{\infty} e^{-\phi_{r}(u, v(u))} h(u) d u,
$$

where $\phi_{r}(u, v)$ is given in (3.2), $v(u)$ in (3.4) and

$$
h(u)=\frac{d t}{d u}=\frac{d[u+i v(u)]}{d u}=1+i \frac{d v(u)}{d u}=1+i \frac{2 u v-y}{v^{2}-u^{2}+x} .
$$

If ph $z=2 \pi / 3$, then $y=-x \sqrt{3}, x \leq 0$, and equation (3.3) can be solved explicitly. The two solutions are

$$
v=u \sqrt{3} \quad \text { and } \quad u=-\frac{3 x+v^{2}}{v \sqrt{3}} .
$$

In this case the path of integration $\mathcal{C}$ runs from the origin to the point

$$
t_{0}=u_{0}+i v_{0}=\sqrt{-x / 2}+i \sqrt{-3 x / 2}
$$

along the line $v=u \sqrt{3}$, and for $u \geq u_{0}$ the path $\mathcal{C}$ follows the hyperbola defined by the second solution given in (3.8); see the right figure in Figure 3.1. The point $t_{0}=u_{0}+i v_{0}=\sqrt{z}=\sqrt{x-i x \sqrt{3}}, x \leq 0$, is a saddle point of the function $\phi(t)$ defined in (3.1).

We can also solve (3.3) for $u$, which gives

$$
u=\frac{y-R}{2 v}, \quad R=\sqrt{y^{2}+4 v^{2}\left(x+\frac{1}{3} v^{2}\right)},
$$

where the square root is nonnegative. This solution should be used for $0 \leq v \leq$ $v_{1}, 0 \leq u \leq u_{1}$, where

$$
v_{1}=v_{1}(x, y)=\sqrt{\frac{3}{2}\left(-x-\sqrt{x^{2}-y^{2} / 3}\right)},
$$

the smallest positive $v$-value for which $R=0$ and $u_{1}=y /\left(2 v_{1}\right)$. For $0 \leq v \leq$ $v_{1}, u \geq u_{1}$, we use $u=(y+R) /(2 v)$; see Figure 3.1.

When integrating with respect to $v$, the integral in (3.6) can be written as

$$
\operatorname{Hi}(z)=\frac{1}{\pi}\left[\int_{0}^{v_{1}} e^{-\phi_{r}\left(u^{-}(v), v\right)} h(v) d v+\int_{v_{1}}^{0} e^{-\phi_{r}\left(u^{+}(v), v\right)} h(v) d v\right],
$$

where $\phi_{r}(u, v)$ is given in (3.2),$u^{ \pm}(v)=(y \pm R) /(2 v)(\mathrm{cf}$. (3.9) $)$ and

$$
h(v)=\frac{d t}{d v}=\frac{d(u+i v)}{d v}=\frac{d u}{d v}+i=\frac{v^{2}-u^{2}+x}{2 u v-y}+i .
$$

Remark 3.1. For the sector $0 \leq \mathrm{ph} z \leq \frac{2}{3} \pi$ we can use a similar method, although we don't need to consider this sector. If $y^{2}-3 x^{2} \geq 0$ the quantity $R$ of (3.9) is defined for all values of $v$, and the first equation in (3.9) defines a path $\mathcal{L}$ going from the origin to $\infty \exp (2 \pi i / 3)$. Because there is a hill at $\infty \exp (\pi i / 3)$, we cannot replace the integration path in (1.4) by $\mathcal{L}$. We need an extra integral from 


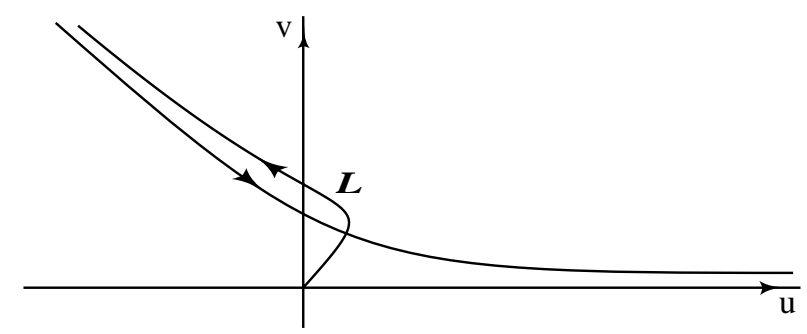

Figure 3.2. The two contours for the integrals described in Remark 3.1 .

$\infty \exp (2 \pi i / 3)$ to $+\infty$, and that integral gives an Airy function; see Figure 3.2. In this way we obtain

$$
\operatorname{Hi}(z)=\frac{1}{\pi} \int_{\mathcal{L}} e^{-\phi_{r}(u, v)} h(v) d v+2 e^{-\pi i / 6} \operatorname{Ai}\left(z e^{-2 \pi i / 3}\right),
$$

with $h(v)$ given in (3.11) and the relation between $u$ and $v$ given in (3.9). We see that the first term in the right-hand side of (2.7) with upper signs corresponds to the integral in (3.12).

Remark 3.2. Comparing the representations of $\operatorname{Hi}(z)$ in (3.6) and (3.12), we observe that the Airy function in (3.12) disappears as $z$ crosses the half-line ph $z=2 \pi / 3$. On that line the argument of the Airy function $\operatorname{Ai}\left(z e^{-2 \pi i / 3}\right)$ becomes positive (see (3.9)). Thus the dominance of $\operatorname{Hi}(z)$ over $\operatorname{Ai}\left(z e^{-2 \pi i / 3}\right)$ is maximal at this line; this is therefore the place where the exponentially-small contribution is "switched on". This aspect is connected with the Stokes phenomenon in asymptotics, and the half-line ph $z=2 \pi / 3$ is a Stokes line for $\operatorname{Hi}(z)$; see [12.

3.1. The Scorer function $\operatorname{Gi}(z)$. It is convenient to have a direct method for $\operatorname{Gi}(z)$ that is not based on results for the $\mathrm{Hi}$-function, especially if $z$ is positive. We give only a few details on this case. The method can be used for the sector $0 \leq$ ph $z \leq 2 \pi / 3$.

The first step is to replace the sine function by two exponentials. Then we obtain

$$
\mathrm{Gi}(z)=\frac{1}{2 \pi i}\left[\mathrm{Gi}^{+}(z)-\mathrm{Gi}^{-}(z)\right], \quad \mathrm{Gi}^{ \pm}(z)=\int_{0}^{\infty} e^{ \pm i \psi(t)} d t
$$

where $\psi(t)=z t+\frac{1}{3} t^{3}=\psi_{r}(u, v)+i \psi_{i}(u, v)$, with

$$
\begin{aligned}
& \psi_{r}(u, v)=\frac{1}{3} u^{3}-u v^{2}+x u-y v, \\
& \psi_{i}(u, v)=u^{2} v-\frac{1}{3} v^{3}+x v+y u .
\end{aligned}
$$

The path of integration for $\mathrm{Gi}^{ \pm}(z)$ is found by solving the equation $\psi_{r}(u, v)=0$. For $\mathrm{Gi}^{+}(z)$ the path runs into the valley at $\infty \exp (\pi i / 6)$. The result is

$$
\mathrm{Gi}^{+}(z)=\int_{0}^{\infty} e^{-\psi_{i}(u, v)} g(u) d u, \quad g(u)=1+i \frac{d v}{d u}=1+i \frac{u^{2}-v^{2}+x}{2 u v+y} .
$$

For $\mathrm{Gi}^{-}(z)$ the integral along $[0, \infty)$ can be replaced by a path along the half-line with ph $t=-\pi / 6$. In this valley no real solution of $\psi_{r}(u, v)=0$ is available, and 
we take a path that first runs into the valley at $\infty \exp (-5 \pi i / 6)$ and then returns to the valley at $\infty \exp (-\pi i / 6)$. This introduces an Airy function, and we obtain

$$
\mathrm{Gi}^{-}(z)=-\int_{0}^{\infty} e^{-\psi_{i}(u, v)} g(u) d u+2 \pi \operatorname{Ai}(z),
$$

where $g(u)$ is as in (3.15). Adding the results in (3.15) and (3.16), we obtain a simple nonoscillating integral plus an Airy function:

$$
\operatorname{Gi}(z)=\frac{1}{\pi i} \int_{0}^{\infty} e^{-\psi_{i}(u, v)} g(u) d u+i \operatorname{Ai}(z),
$$

where the relation between $u$ and $v$ is given by

$$
v=\frac{-y+\sqrt{y^{2}+4 u^{2}\left(x+\frac{1}{3} u^{2}\right)}}{2 u} .
$$

If $z$ is real and nonnegative, (3.17) becomes real. The term with the Airy function is canceled by the imaginary contribution of $g(u) / i$. The remaining integral in (3.17) should be modified in this case. The contour runs from the origin to the saddle point $i \sqrt{x}$, and from this point into the valley at $\infty \exp (\pi i / 6)$. Integrating with respect to $v$, we obtain the real representation

$$
\operatorname{Gi}(x)=\frac{1}{\pi}\left[\int_{0}^{\sqrt{x}} e^{-x v+\frac{1}{3} v^{3}} d v+\int_{\sqrt{x}}^{\infty} e^{2 x v-\frac{8}{3} v^{3}} d v\right], \quad x \geq 0 .
$$

\section{NumericAl ILLUSTRATIONS}

We give some numerical results which serve as demonstration of our method.

In order to evaluate the Scorer functions in the whole complex plane, we need to select software for the evaluation of Airy functions of complex arguments and for the quadrature of real functions over an infinite integral. For the first purpose, we use the public domain subroutines ZAIRY and ZBIRY by D.E. Amos [2], and for the semi-infinite integral we use the automatic adaptative integrator DQAGIE by R. Piessens. All these codes can be retrieved from the SLATEC public domain library [13] (see also GAMS: guide to available mathematical software [6]).

The connection formulae given in Section 2 together with the nonoscillating integrals derived in Section 3 can be used to evaluate $\mathrm{Hi}(z)$ and $\operatorname{Gi}(z)$ in the whole complex plane. By using the integral representations for $\operatorname{Hi}(z)$ in the domain $\pi \leq$ ph $z \leq 2 \pi / 3$ the following stable algorithm can be considered:

Algorithm 4.1 (via (3.6) $)$.

- Whenever $\operatorname{Im}(z)<0$, use (2.9)).

- If $z \in S_{1}^{(2)}$, obtain $\mathrm{Hi}(z)$ by quadrature.

- If $z \in S_{1}^{(1)} \cup S_{0}$, obtain $\mathrm{Hi}(z)$ via (2.7).

- Obtain $\operatorname{Gi}(z)$ everywhere in the complex plane by using (2.8).

where $S_{1}^{(1)}$ is the sector $\pi / 3 \leq \mathrm{ph} z \leq 2 \pi / 3$ and $S_{1}^{(2)}$ is the sector $2 \pi / 3 \leq \mathrm{ph} z \leq \pi$.

However, in the fourth step two integrals for Hi will be needed. Thus, the following stable scheme is expected to be more efficient when fast algorithms to compute the Airy functions $\mathrm{Ai}$ and $\mathrm{Bi}$ are available: 
TABLE 4.1. The real and imaginary parts of $\operatorname{Hi}(z)$ in the sector $\pi \leq \mathrm{ph} z \leq \frac{2}{3} \pi$. The result from the asymptotic expansion up to order $1 / z^{10}$ is shown inside brackets. The number of integration steps for each evaluation is shown within the braces.

\begin{tabular}{|l|l|l|l|l|}
\hline & & ph $z=\pi$ & ph $z=5 \pi / 6$ & ph $z=2 \pi / 3$ \\
\hline$|z|=1$ & Re (Hi) & 0.22066961 & 0.22331566 & 0.23477589 \\
& & $\{195\}$ & $\{195\}$ & $\{345\}$ \\
\hline$|z|=1$ & Im (Hi) & 0 & $6.213302110^{-2}$ & 0.13605894 \\
& & & $\{165\}$ & $\{465\}$ \\
\hline \hline$|z|=10$ & Re (Hi) & $3.176853510^{-2}$ & $2.759714510^{-2}$ & $1.594800310^{-2}$ \\
& & $\{75\}$ & $\{75\}$ & $\{135\}$ \\
& & $\left(3.176852810^{-2}\right)$ & $\left(2.759713710^{-2}\right)$ & $\left(1.594799810^{-2}\right)$ \\
\hline$|z|=10$ & Im (Hi) & 0 & $1.585978910^{-2}$ & $2.762275110^{-2}$ \\
& & & $\{75\}$ & $\{225\}$ \\
& & & $\left(1.585978610^{-2}\right)$ & $\left(2.762274210^{-2}\right)$ \\
\hline \hline$|z|=100$ & Re (Hi) & $3.183092510^{-3}$ & $2.756647710^{-3}$ & $1.591552610^{-3}$ \\
& & $\{135\}$ & $\{165\}$ & $\{165\}$ \\
& & $\left(3.183092510^{-3}\right)$ & $\left(2.756647710^{-3}\right)$ & $\left(1.591552610^{-3}\right)$ \\
\hline$|z|=100$ & Im (Hi) & 0 & $1.591543910^{-3}$ & $2.756650010^{-3}$ \\
& & & $\{165\}$ & $\{165\}$ \\
& & & $\left(1.591543910^{-3}\right)$ & $\left(2.756650010^{-3}\right)$ \\
\hline
\end{tabular}

Algorithm 4.2 (via (3.6) and (3.17)).

- Whenever $\operatorname{Im}(z)<0$, use (2.9).

- If $z \in S_{1}^{(2)}$, obtain $\mathrm{Hi}(z)$ by quadrature.

- If $z \in S_{1}^{(1)} \cup S_{0}$, obtain $\mathrm{Gi}(z)$ by quadrature via (3.17) or (3.18).

- For the remaining cases, apply (1.6).

The second algorithm is preferred in most circumstances. However, we have experienced that the computation of $\operatorname{Gi}(z)$ when $\operatorname{Re}(z)>0$ and $\operatorname{Im}(z) \rightarrow 0$ is more efficient when the first algorithm is considered. Probably, the best numerical strategy is a combination of both algorithms, together with the use of asymptotic expansions for large $|z|$ and series expansions for small $|z|$. The best strategy may also depend on the choice of the quadrature rule. This numerical study lies beyond the scope of the present paper.

We end this section by showing numerical results. Of interest are the sectors where (2.5) and (2.6) are valid, namely, $S_{1}^{(2)}$ for $\operatorname{Hi}(z)$ and $S_{1}^{(1)} \cup \mathcal{S}_{0}$ for $\operatorname{Gi}(z)$. Of particular interest are the regions $S_{0}$ for $\operatorname{Gi}(z)$ and $S_{1}^{(2)}$ for $\operatorname{Hi}(z)$, since in this case we compute the functions directly by quadrature, and the corresponding integrals can be compared with asymptotics if $z$ is large. We are giving explicit results with an accuracy of 8 digits for $\mathrm{Hi}(z)$ which are compared with the asymptotic expantion (2.5) up to order $1 / z^{10}$ whenever this is possible.

The results in Table 4.1 are obtained by means of a Fortran program coded in double precision arithmetic in which the integral (3.6) is evaluated. Together with the results, we show the number of integration steps needed to attain an accuracy of 8 digits. The results from asymptotics (shown inside parentheses) are seen to coincide with those from the integral (3.6) for $|z|=100$, but for $|z|=10$ we 

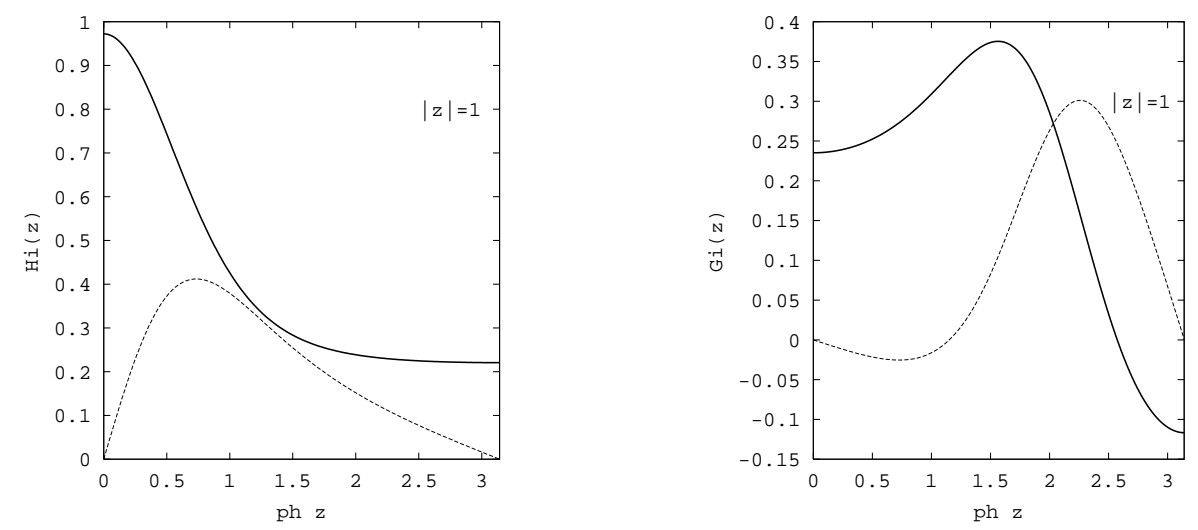

Figure 4.2. The real (solid) and imaginary (dashed) parts of $\operatorname{Hi}(z)$ (left) and $\operatorname{Gi}(z)$ (right) for $|z|=1$ and $0 \leq \operatorname{ph~} z \leq \pi$.

observe discrepancies in the last digits which are due to the failure of the asymptotic expansion ( $z$ is not large enough). We also found agreement with the asymptotic expansion for $\operatorname{Hi}(z)$ for large $z$ in the sector $S_{1}^{(2)}$. For real negative $x$ our results coincide with those given by Scorer [15].

One sees that at the Stokes line ph $z=2 \pi / 3$ the quadrature requires more steps, as can be expected given the appearance of a discontinuity in $d v / d u$ at the maximum $v$. On the other hand, the faster convergence takes place when we are far from the Stokes line. Moreover, convergence tends to be slower as $|z|$ becomes smaller; as $|z|$ becomes larger the effect of the singularity in the derivative $d v / d u$ for ph $z=\frac{2}{3} \pi$ would appear at larger $u$, where the exponential in the integrand is smaller.

Similarly, one can test the performance of the integral representation (plus an Airy function) for $\operatorname{Gi}(z)$ in the sector $0 \leq \operatorname{ph} z \leq 2 \pi / 3$, which should coincide with the results from the asymptotic expansion (2.6) for $|\mathrm{ph} z|<\pi / 3$ and large $z$. We also find agreement with the results tabulated by Scorer for real positive $x$. One observes that the convergence of the integral representation (3.17) becomes slower as we approach the real line ph $z=0$. With the quadrature DQAGIE we obtain a better performance using Algorithm 4.1 in this case.

As a further illustration, in Figure 4.2 we give two plots for $|z|=1$ obtained by using Algorithm 4.2. The graphs show the real and imaginary parts of the Scorer functions. One observes the smooth connection between the different sectors in the complex plane.

\section{Summary AND CONCLUding REMARKS}

As mentioned in the cited references (see [7] and [9]), the inhomogeneous Airy functions (Scorer functions) are used in several physical problems. We have used functional relations and derived integral representations of the Scorer functions that can be used for stable numerical computations for all complex values of the $\operatorname{argument} z$. The integrals can be easily split up into real and imaginary parts.

For the Scorer functions, Maclaurin and asymptotic expansions are available. To bridge the gap between the areas where convergent or asymptotic series can be 
used, one can use the representations in this paper, although they can be used for all values of the argument. The tool one needs is a suitable quadrature method for computing real integrals on unbounded real intervals that converge very fast at infinity. We have illustrated the method by giving a few numerical results based on selecting a quadrature rule; we have not investigated an optimal choice of quadrature rule for computing the Scorer functions.

We have shown how to handle oscillating integrals for a certain set of special functions, and this is quite instructive for applying the method to other functions. A similar method can be used for the Airy functions, and another treatment can be found in [16] for modified Bessel functions of imaginary order. Still, many special functions need to be considered in order to get reliable software, in particular for complex and/or large values of the parameters.

\section{ACKNOWLEDGMENTS}

A.G. and J.S. would like to acknowledge the hospitality of CWI during their stay. A.G. and J.S. also acknowledge financial support from the Consellería de Educación y Ciencia (Generalitat Valenciana).

The authors thank the referee for valuable suggestions.

\section{REFERENCES}

1. M. Abramowitz and I.A. Stegun (Eds.), Handbook of Mathematical functions, National Bureau of Standards Applied Mathematics Series No. 55. U.S. Government Printing Office, Washington, DC; many editions. MR 29:4914 MR 94b:00012 etc.

2. D.E. Amos. "Algorithm 644: A portable package for Bessel functions of a complex argument and nonnegative order". ACM Trans. Math. Softw. 12 (1986) 265-273. CMP 19:12

3. R.M. Corless, D.J. Jeffrey and H. Rasmussen "Numerical evaluation of Airy functions with complex arguments". J. Comput. Phys. 99 (1992), 106-114. MR 92k:65028

4. H. Exton. "The asymptotic behaviour of the inhomogeneous Airy function $\operatorname{Hi}(z)$ ". Math. Chronicle 12 (1983), 99-104. MR 84g:33004

5. B. Fabijonas "The computation of Scorer functions". Lecture during the 1998 Annual SIAM Meeting in Toronto, Canada.

6. GAMS: Guide to available mathematical software. http://gams.nist.gov

7. S.-Y. Lee "The inhomogeneous Airy functions, $\mathrm{Gi}(z)$ and $\mathrm{Hi}(z)$ ". J. Chem. Phys. 72 (1980), 332-336. MR 81b:33015

8. D.W. Lozier and F.W.J. Olver. "Numerical evaluation of special functions". In W. Gautschi (Ed.), AMS Proceedings of Symposia in Applied Mathematics 48 (1998), pp. 79-125. MR 95m:65036

9. A.J. MacLeod. "Computation of inhomogeneous Airy functions". J. Comput. Appl. Math. 53 (1994) 109-116. MR 95k:65023

10. The National Institute of Standards and Technology has a public web site that includes an extensive treatment of Scorer functions: http://www.nist.gov/DigitalMathLib.

11. F.W.J. Olver. Asymptotics and Special Functions. Academic Press, New York. Reprinted in 1997 by A.K. Peters. MR 55:8655, MR 97i:41001

12. R.B. Paris and A.D. Wood. "Stokes phenomenon demystified", IMA Bulletin 31 (1995) No.12,21-28. CMP 95:10

13. SLATEC Public Domain Mathematical Library. gopher://archives.math.utk.edu/11/software/multi-platform/SLATEC

14. Z. Schulten, D.G.M. Anderson, and R.G. Gordon. "An algorithm for the evaluation of complex Airy functions". J. Comput. Phys. 31 (1979) 60-75. MR 80c:65043

15. R.S. Scorer. "Numerical evaluation of integrals of the form $I=\int_{x_{1}}^{x_{2}} f(x) e^{i \phi(x)} d x$ and the tabulation of the function $\operatorname{Gi}(z)=(1 / \pi) \int_{0}^{\infty} \sin \left(u z+\frac{1}{3} u^{3}\right) d u$ ". Quart. J. Mech. Appl. Math. 3 (1950) 107-112. MR 12:287c

16. N.M. Temme. "Steepest descent paths for integrals defining the modified Bessel functions of imaginary order". Methods Appl. Anal. 1 (1994) 14-24. MR 95g:33006 
17. N.M. Temme. Special functions: An introduction to the classical functions of mathematical physics. John Wiley \& Sons, New York, 1996. MR 97e:33002

18. R. Wong Asymptotic approximations of integrals. Academic Press, New York, 1989. MR 90j:41061

Instituto de Bioingeniería, Universidad Miguel Hernández, Edificio La Galia. 03202Elche (Alicante), Spain

E-mail address: amparo@titan.ific.uv.es

Instituto de Bioingeniería, Universidad Miguel Hernández, Edificio La Galia. 03202Elche (Alicante), Spain

E-mail address: segura@flamenco.ific.uv.es

CWI, P.O. Box 94079, 1090 GB Amsterdam, The Netherlands

E-mail address: nicot@cwi.n] 